\title{
DESIGNING A THREE-DIMENSIONAL WOVEN FABRIC STRUCTURE AS AN ELEMENT OF A BABY STROLLER
}

\author{
Bethalihem T. Samuel ${ }^{1,2}$, Marcin Barburski ${ }^{1, *}$, Tsegaye Sh. Lemmi $^{1}$ \\ 1 Faculty of Material Technologies and Textile Design, Institute of Architecture of Textile, Lodz University of Technology, 116 Zeromskiego Street, \\ 90-924 Lodz, Poland \\ 2 Department of Textile Engineering, Kombolcha Institute of Technology, Wollo University, Ethiopia \\ ${ }^{*}$ Corresponding author. E-mail: marcin.barburski@p.lodz.pl
}

\begin{abstract}
:
A baby stroller allows the transportation of a child over long or short distances. The materials used to produce the stroller make it heavy for users, which creates difficulties when lifting the stroller. The goal of this project was to design and fabricate a three-dimensional (3D) fabric structure that can be used as part of a stroller seat to improve its mechanical and physical properties. The idea of implementing a woven 3D system allows the development of an egg-shaped or shell-like structure as part of a stroller seat. The combination of double-woven material and honeycomb polypropylene (as the reinforcing material) was used to create a 3D composite structure. Single and double layers of polypropylene honeycomb sandwiched within layers of linen flax fabric were used to prepare the composite samples. Subsequently, tests on mechanical and physical properties, such as density, flexural strength, and tensile strength, were carried out. Analysis of the results showed that the composite with one layer of honeycomb has half the density of polyvinyl chloride.
\end{abstract}

\section{Keywords:}

Composite, bending, tensile, woven fabric, flax fiber, honeycomb, stroller

\section{Introduction}

The manufacture of $3 D$ textile weaves started around the year 1980 [1]. The yarns were arranged in three mutually perpendicular directions, i.e., in the directions of vertically $(z)$, longitudinally $(x)$, and cross-wisely $(y)$ intermeshed fibers, to form a 3D textile woven fabric. This kind of interlacement forms a complex integrated structure. A 3D weave structure helps to increase the mechanical performance, such as shear resistance, and facilitates low thermal distribution across the material equally in the three directions [2-4]. Currently, the enormous growth of industries and engineering applications has enabled the development of innovations in 3D textile and composite technologies and has started a new chapter by extending the usage and application areas. The manufacturing process has many steps, which start from the processing of different kinds of raw fibers to the end of the structure formation using weaving, knitting, nonwoven, and braided manufacturing methods. The 3D woven fabric can be produced by conventional weaving technology and multiwarp weaving technology. The latter technology is capable of constructing different types of geometrical shapes of $3 D$ fabrics, such as fully interlaced weave, orthogonal weave, and angle interlock weave. In addition, the geometrical shapes depend on the yarn and the production parameters [5-7].

Textile-based composites are formed from one or more layers of fabric by immersing it into a resin system [8]. The composite engineering lamination of $3 D$ fabrics by using $2 D$ fabrics gives an advantage by increasing the strength and the thickness of the material. The woven composite is formed from three main components, namely, warp yarn, weft yarn, and matrix resin. The combination of these creates a single composite with excellent tribological properties. Moreover, using woven composites, one can also easily manufacture a complexshaped material, which can be used in many industries [9-11]. During the fabrication of complex shapes, the main requirement is avoiding the wrinkling of the reinforcement material during composite preparation because wrinkling decreases the mechanical quality during various applications [12].

The use of textile-reinforced composite with woven, knitted, and braided fabrics has widely increased in various industries, such as aerospace technology, reinforcement applications, geotextiles, protective clothes, and other sectors [13], due to their unique properties, such as mechanical strength, fatigue resistance, light weight, thermal resistance, chemical resistance, corrosion resistance, and so on [2, 3]. The mechanical properties of textile composites depend on the fiber's type, fabric architecture, and matrix properties [8].

A stroller (referred to as pushchair/buggy in commonwealth countries) is a three- or four-wheeled manual vehicle used for transporting a baby or small child, who is not capable of traveling long distances on his/her own. The history of strollers tells us that the primary materials used for the construction of 
stroller were wood and steel materials; however, nowadays, stroller parts are specifically made from aluminum for the frame, textile fabrics for the seat and covering, and rubber and plastics for handles, as well as wheels [14]. The purpose of this work was to design a shell-like shape using 3D woven fabric and to construct a $3 D$ composite as an element of a baby stroller part. The 3D woven composite used for a stroller seat (base) increases the mechanical and physical properties, which means that it improves the lightweight property and life span of the material.

\section{Materials and methods}

The materials used for the weaving of double cloth were flax yarn (190 tex), which was used as the weft yarn, and polyester (polyethylene terephthalate [PET] 165 dtex) fiber as the warp yarn. As a result, the average single-strand flax yarn can resist a force $12.76 \mathrm{~N} /$ tex before breaking point, and the maximum elongation at break is $1.24 \%$.

Using natural fibers such as flax as a reinforcement material has several advantages, such as favorable mechanical properties and good acoustics; moreover, the production energy of natural fibers is much lower than that of synthetic fibers. The main disadvantages of natural fiber are its high moisture absorption capacity and the level of compatibility between the matrix and fabric during composite formation [15].

Additionally, the material used for composite formation was polypropylene (PP) honeycomb (Coremat XM, with a thickness of $2 \mathrm{~mm}$ ), made from plastic material, which is an ideal component for the production of a sandwich composite. One of the advantages of using a PP honeycomb as a sandwich is

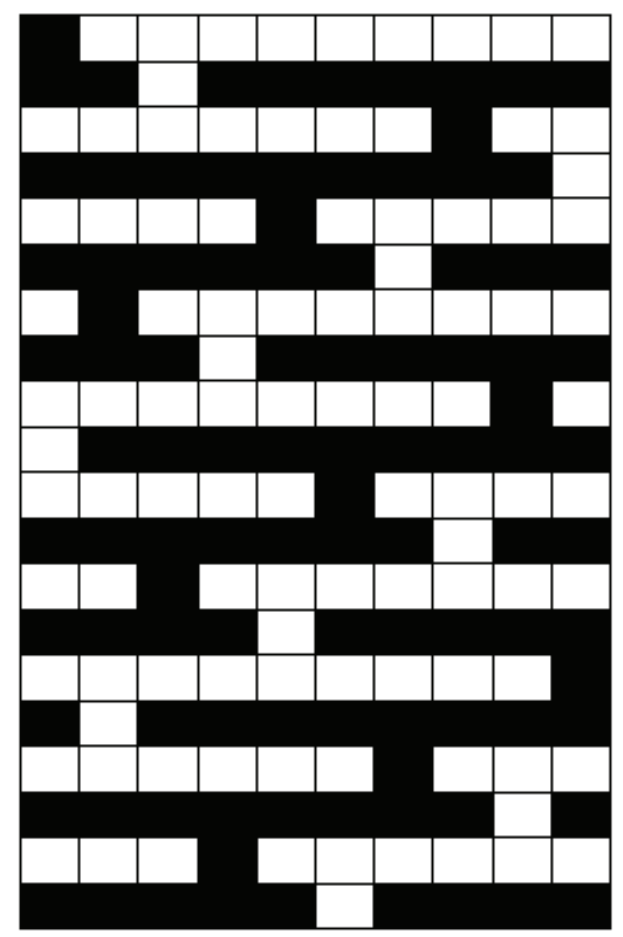

a. Stitched part of 3D double woven fabric the resulting increase in the thickness of the composite. The PP honeycomb allows the final product to have the following features: durability, lightweight property, high tensile strength, high bending strength, and pressure resistance [16].

\subsection{Design of 3D shell structure}

The intent of creating a shell structure was to form the seat part of the baby stroller. Assembly of the 3D shell structure was done by using a double-woven fabric manufacturing system, and finally, using a double-woven flax fabric as a reinforcement material, a 3D composite structure was created.

\subsection{Manufacture of 3D fabric}

Self-stitched double-cloth fabrics were woven using a Picanol Gamma Jacquard Machine J41010/T6-32. A sateen weave $(1 / 4(2)$ ) (in a single layer of a double fabric) was formed by using flax yarn 190 tex as a weft and polyester (PET $165 \mathrm{dtex}$ ) as a warp yarn with dimensions of weaving $40 \mathrm{~cm} \times 40 \mathrm{~cm}$ (Figure 2).

\subsection{Flat composite sample preparation}

The composite preparation step is necessary in this project to increase the strength of the woven fabric by changing it to a stiff structure and, at the same time, by adding to the thickness of the fabric layer without weight gain of the structure. The composites were made using a vacuum bagging method. This method enhances the required shape by squeezing the fabric layers into the shape of the mold surface due to the pressure of the pump. The main advantage of vacuum bagging is that a resin can flow throughout the surface due to the applied pressure, and this can prevent the early failure of the parts

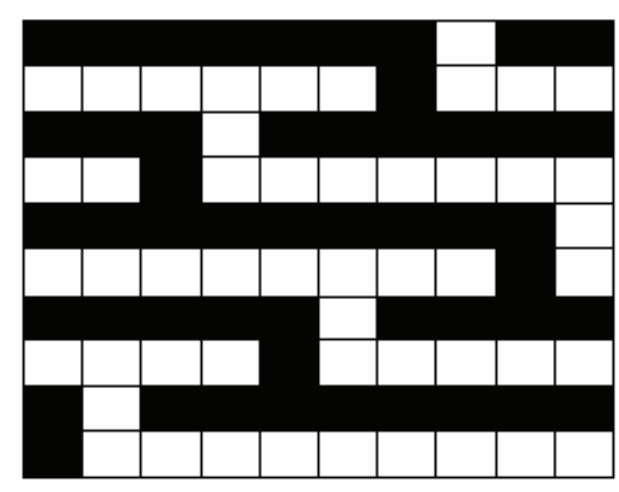

b. Open part of 3D double woven fabric

Figure 1. A schematic internal unit cell of the 3D double-woven structure. 


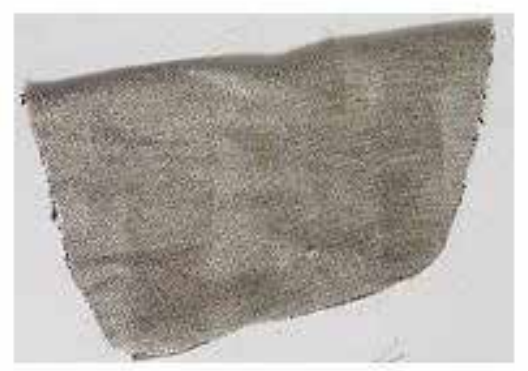

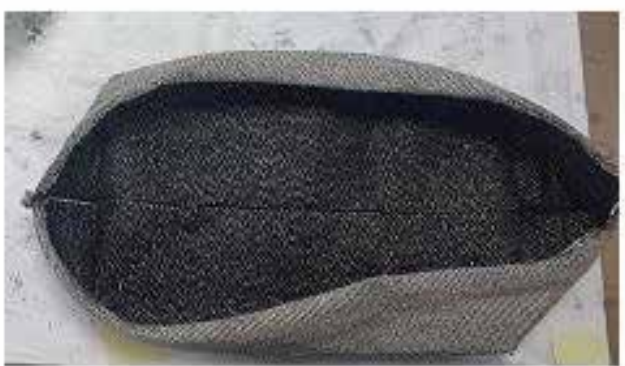

b. Top view of the fabric

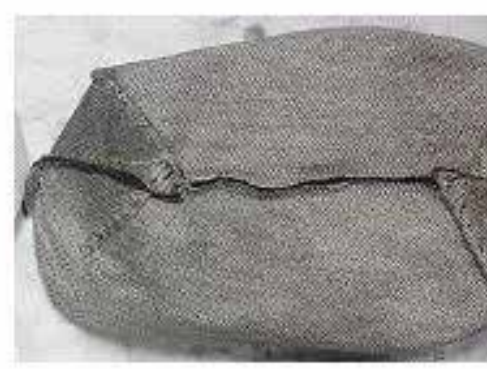

c. Back view of the fabr

\section{a. Front view of the fabric.}

Figure 2. Three-dimensional (3D) views of the woven fabric from different directions.

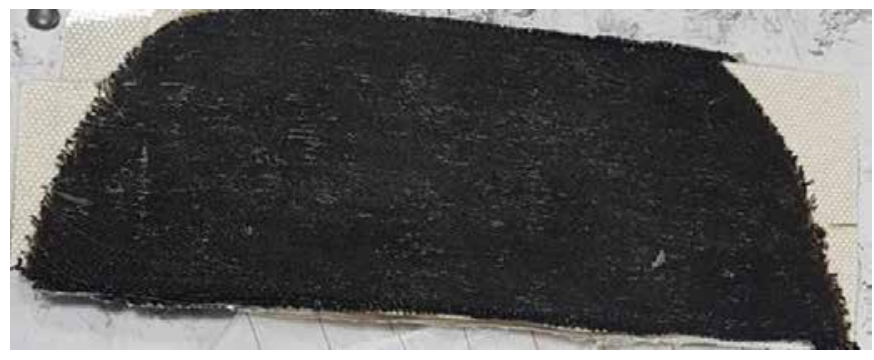

Figure 3. Flat composite sample.

[4, 17-19]. The prepared samples are of two kinds. The first one contains two layers of woven fabric and one layer of PP honeycomb between the two layers of fabrics.

The second sample contains two layers of woven fabric and two layers of honeycomb between the two layers of fabrics, after manual impregnation of each flax fabric and honeycomb on both sides using epoxy resin.

Subsequently, the materials are laid on a previously prepared surface as the single- or double-layered honeycomb sandwiched between two layers of flax fabric. Finally, the surface is covered with a plastic vacuum bag and sealed using tape to conveniently suck out air and unnecessary resin from inside the vacuum bag using a 1-bar pressure pump (Figure 3).

\subsection{Experiment}

The result of testing for the mechanical properties will answer the next two main questions: will this material have the performance needed to use it as a stroller part? Can it replace other synthetic materials that are used for the production of a stroller? To answer these two questions and for comparison of their mechanical properties, tensile and bending test experiments were performed on the flat samples. Both experiments were conducted in the Faculty of Mechanical Engineering at the Lodz University of Technology.

\subsubsection{Bending test}

The project's aim was to produce a material of high strength with lightweight properties. The question on how much load can be resisted during the holding of a baby can be answered

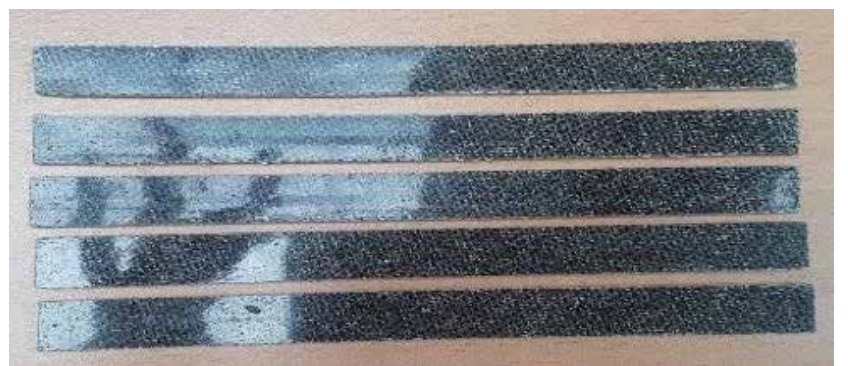

Figure 4. Prepared samples for bending test in the weft and warp directions.

by finding out the flexural strength properties of the prepared composite. The evaluations of the composite samples were carried out using a three-point flexural test in accordance with the norm, viz., British Standards Institute/Europaische Norm/ International Organization for Standardization (BS EN ISO) 14125:1998.

2.4.1.1. Determination of flexural properties of fiber-reinforced plastic composites

The two sample composites prepared were tested separately to find out the effect of the honeycomb on the mechanical performance of the composites. For the two different samples, five test specimens for each direction were prepared, i.e., at $0^{\circ}$ to the flax fiber (weft) and at $90^{\circ}$ to the polyester fiber (warp) directions (Figure 4).

During the experiment, the prepared sample was fixed on the Shimadzu bending machine, and the test was carried out at a constant speed of $5 \mathrm{~mm} / \mathrm{min}$ until the deformation values reached the desired point. The evaluation determines the modulus of elasticity, maximum stress, and flexural point (deformation) (Figure 5 ).

\subsubsection{Tensile test}

Tensile tests of the flax woven fabric and honeycomb composite material were carried out using a Shimadzu tensile machine and conformed to the requirement of the American Society for Testing and Materials (ASTM) D3039; the samples were prepared and placed for the test. The samples were prepared five from each direction and for each type of specimen. During 

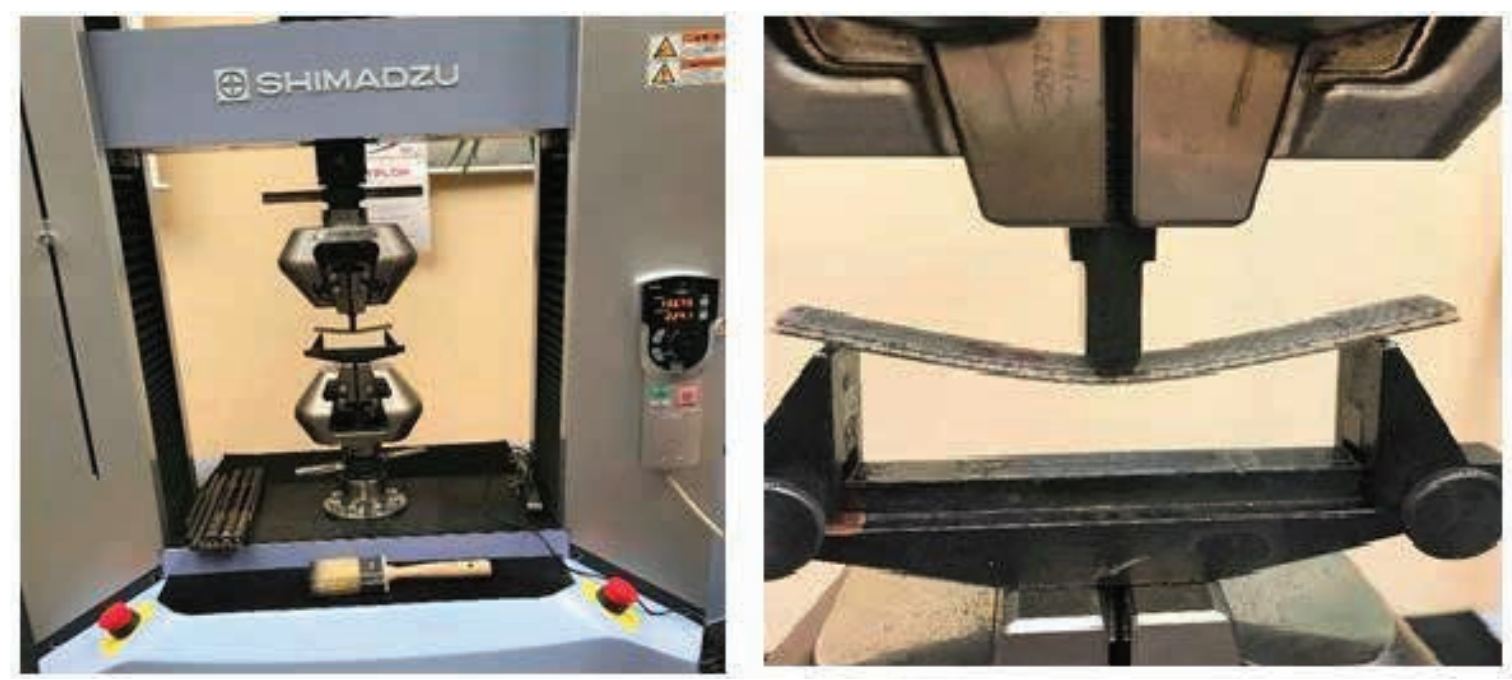

Figure 5. (a) Set up of machine; and (b) flexural testing status.

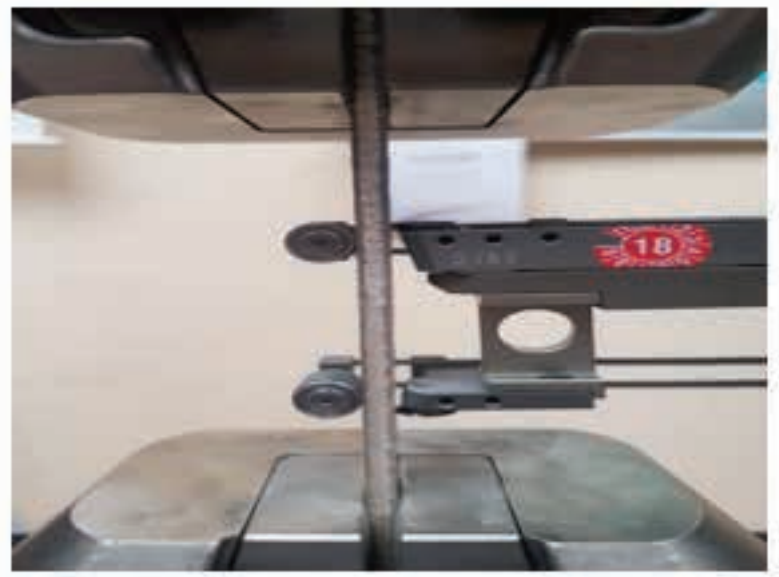

a

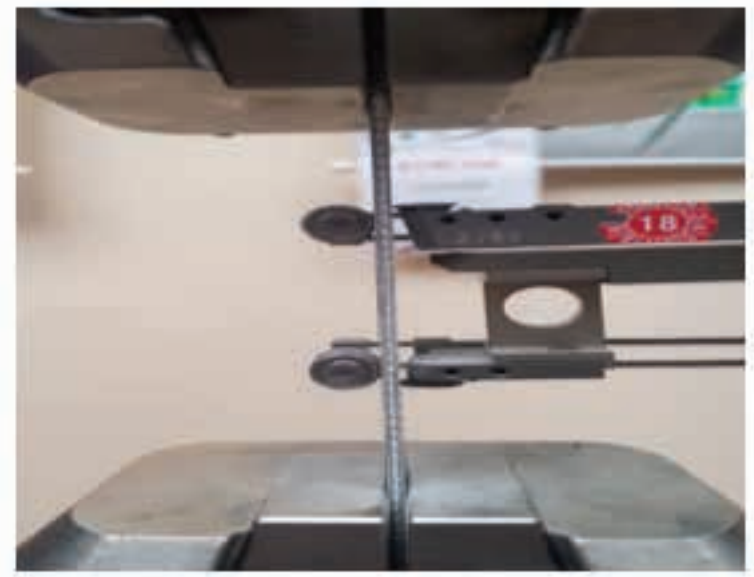

b

Figure 6. Tensile test of (a) double-honeycomb; and (b) single-honeycomb composite samples.

the tensile test, the elongation of the composite was measured using an extensometer (Figure 6).

\section{Results and discussion}

\subsection{Bending test result}

The comparison and the difference in flexural strength between the double PP and single PP honeycomb are displayed clearly in Figure 8. Based on the bending test result, the influence of flax fiber on a single- and double-honeycomb composite in the $0^{\circ}$ direction produces better strength. It is In the single PP sample, in the $0^{\circ}$ direction, the bending strength is higher by $71.74 \%$ than in the $90^{\circ}$ direction; in the double PP sample also, in the $0^{\circ}$ direction, the strength is higher by $61.02 \%$ compared with that in the $90^{\circ}$ direction. This indicates that the resistance of flax yarns in the weft direction to the applied stress force is the maximum; or in other words, the strength of these composites depends on the flax fiber.

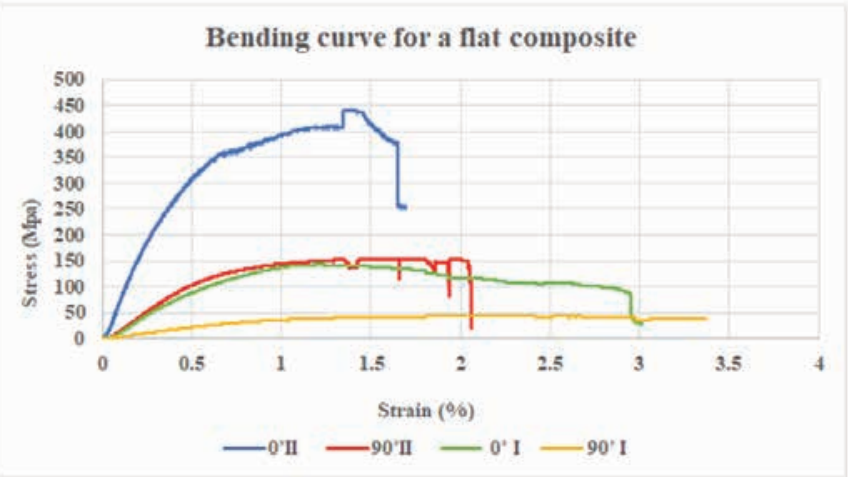

Figure 7. Flexural stress-strain curves for double- and singlehoneycomb PP composite in the $0^{\circ}$ and $90^{\circ}$ directions.

The comparison of the bending test results of the two samples shows that bending strength of the double-PP honeycomb is $32.85 \%$ higher than that of the single-PP honeycomb in the $0^{\circ}$ direction, and the double-PP honeycomb shows $51.37 \%$ higher strength than the single-PP honeycomb in the $90^{\circ}$ direction. 
Moreover, as we can see in Figure 7, the flexural stress-strain curves were plotted from the measured load versus extension values.

This figure gave similar information by strengthening the description of the above test. Therefore, in both directions, the sample that contains double-PP honeycomb shows better flexural stress-strain result than the single-PP honeycomb flat composite sample. Generally, as seen in Figures 7 and 8 , and described earlier, the sample that contains double-PP honeycomb does have better flexural strength due to three main reasons. The first one is that when a force is applied to the hexagonal honeycomb structure, it shows different properties in different directions. In this case, the bending force is applied perpendicular to the sandwiched hexagonal PP honeycomb layer, and this phenomenon increases the resistance of bending strength. The second reason is that as the thickness of the composite increases due to the double-PP honeycomb, the resistance of flexural strength also increases in parallel. The third reason is that due to the presence of the flax yarn, when the force is exerted in the direction of the flax fiber at $0^{\circ}$, the flexural strength of the material also increases.

\subsection{Tensile test result}

The comparison and the difference in tensile test results between the double-PP and single-PP honeycomb are presented in Figure 10. The tensile test result shows that both single- and double- honeycomb composite samples have better tensile strength in the weft direction based on the flax yarn. In the single-PP sample, in the $0^{\circ}$ direction, the tensile strength is higher by $86.51 \%$ than that in the $90^{\circ}$ direction; similarly, in the double-PP sample, in the $0^{\circ}$ direction, the resistance is higher by $74.56 \%$ than that in the $90^{\circ}$ direction; this indicates that the strength of the composite depends on the flax fiber. The comparison of the results from the two samples shows that the single-PP composite has higher strength (by $57.62 \%$ ) than the double-PP composite sample in the $0^{\circ}$ direction, and

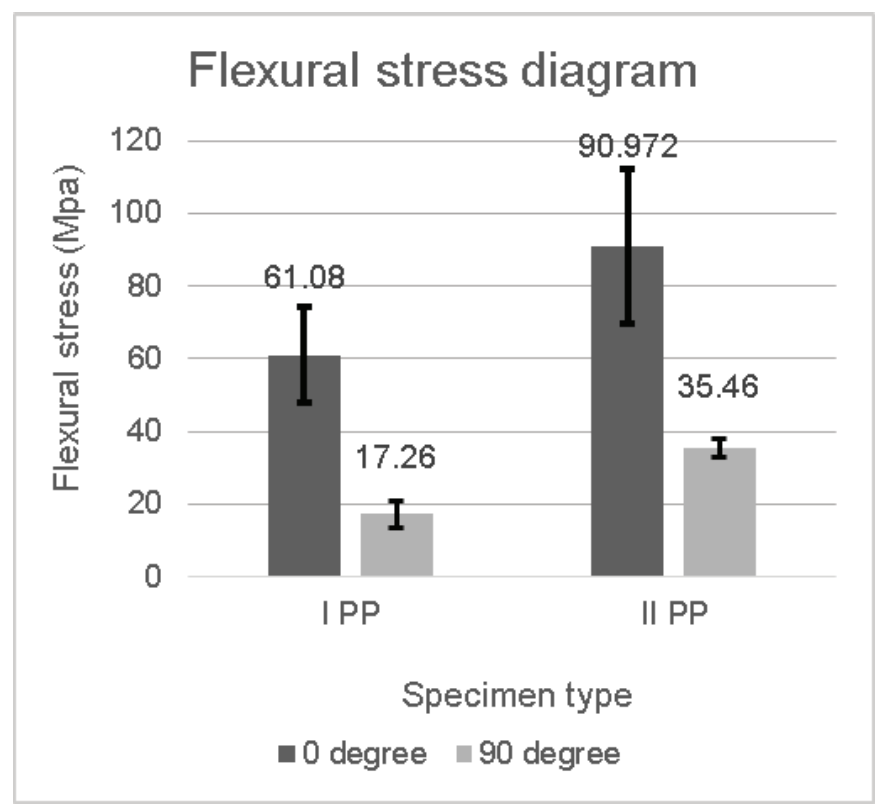

Figure 8. Flexural stress results. that single-PP sample is $20.08 \%$ stronger than the doublePP sample in the $90^{\circ}$ direction. Simultaneously, Figure 9, presenting the tensile stress-strain curves, shows that the single-PP flat composite samples have higher tensile stressstrain values in both directions than do the double-PP composite samples. This result gives additional information on the hereingiven explanations. Generally, the single-PP honeycomb sample does have better tensile strength than the double-PP honeycomb. Because when the tension force is applied in a direction parallel to the sandwiched hexagonal PP honeycomb layer, the strength of the hexagonal PP honeycomb decreases. Moreover, the double layer of PP affects the strength of the flax fabric due to its low range of breakage during the tensile test. In this case, in the $0^{\circ}$ direction, the result from the doublePP honeycomb sample shows two times lower value than that from the single-PP honeycomb. In other words, the single-PP honeycomb sample shows higher tensile strength since the composite has only a single PP honeycomb layer with two layers of flax fabric.

The overall analysis shows that the flax fabric is the main reason for the increasing tensile strength performance in the $0^{\circ}$ direction of a single-PP honeycomb sample.

The results of the elongation test (Figure 11) performed on the single-PP sample in the $90^{\circ}$ (warp) direction show that the elongation is higher by $92.1 \%$ than that in the $0^{\circ}$ (weft)

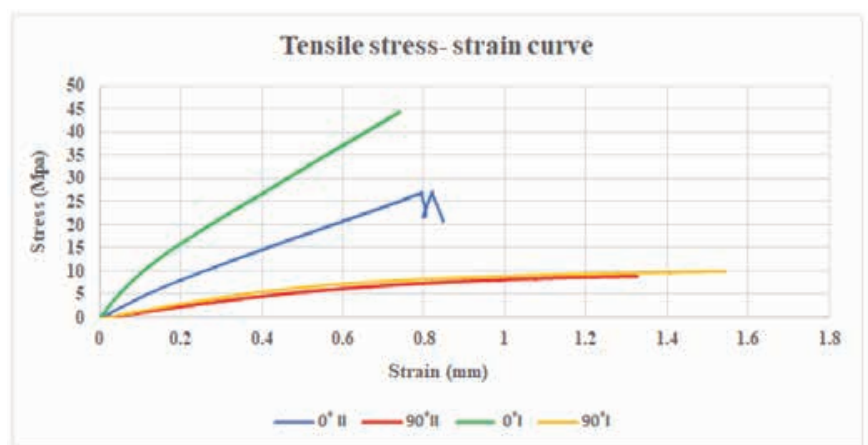

Figure 9.Tensile stress-strain curves for double- and singlehoneycomb PP composites in the $0^{\circ}$ and $90^{\circ}$ directions.

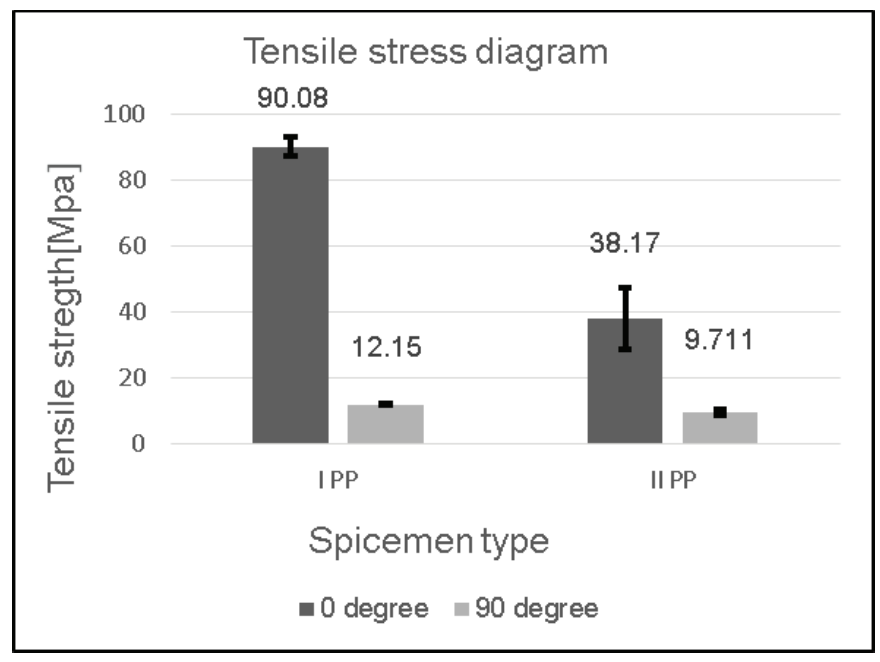

Figure 10. Tensile stress results. 
direction. Moreover, in the double-PP sample, in the $90^{\circ}$ (warp) direction, the elongation of the sample is higher by $85.1 \%$ than that in the $0^{\circ}$ (weft) direction.

This analysis indicates that the elongation of the polyethylene $(P E)$ fiber is higher than that of the flax fiber. Moreover, the comparison between the single-PP sample and the double-PP sample in the $0^{\circ}$ direction shows that the single-PP sample has higher elongation (by $52.5 \%$ ) than the double-PP sample. In addition, in the $90^{\circ}$ direction, the single-PP sample shows higher elongation (by $72.75 \%$ ) than the double-PP sample. Generally, the warp direction, which has PE fibers, has a higher elongation value than the weft direction, containing flax fibers. This phenomenon indicates that for the production of stroller material, PE is not convenient due to its high elongation properties.

The physical properties can describe the weightiness of a material. Therefore, as the density decreases, the weightlessness of the material becomes much better. From the

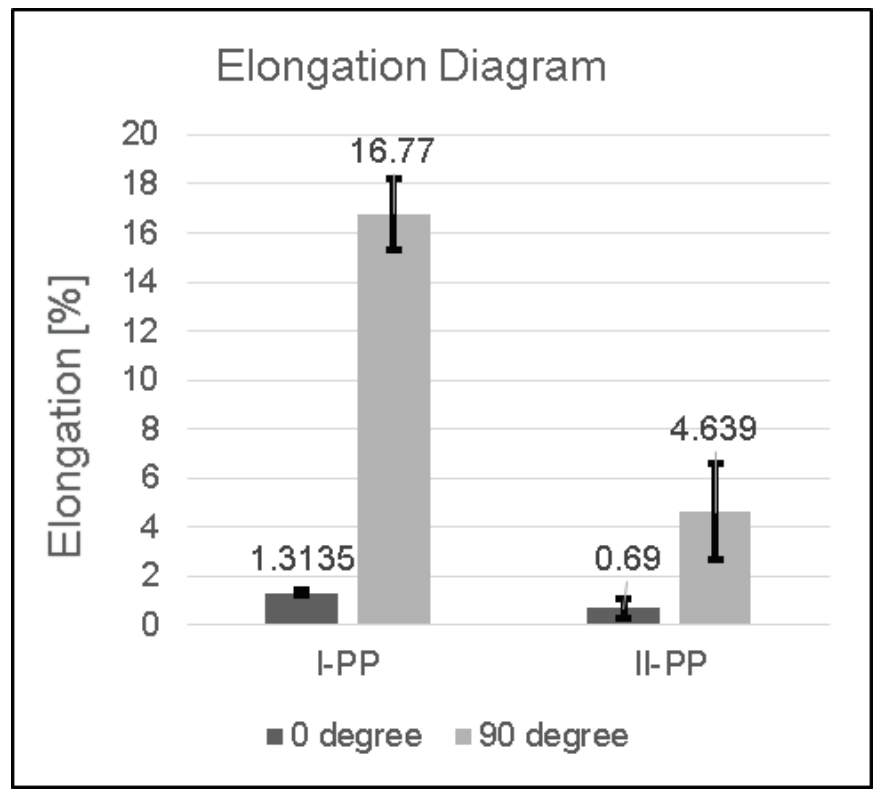

Figure 11. Elongation result based on the tensile test of a flat composite sample.

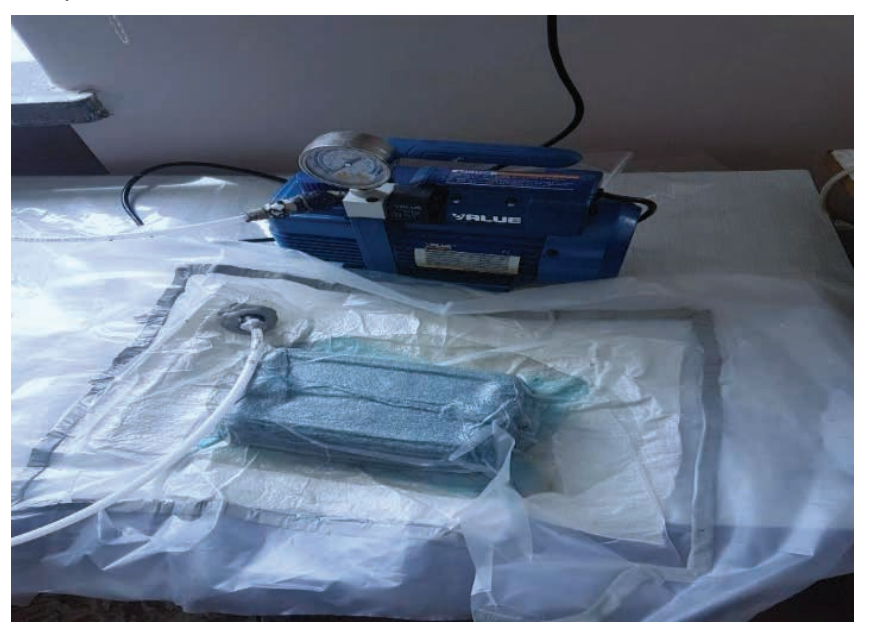

Figure 12. Composite prototype formation using the vacuum bagging method. test results, the density of a sample that consists of singlePP honeycomb is $0.67 \mathrm{~g} / \mathrm{cm}^{3}$, and a sample with double-PP honeycomb has a density of $0.615 \mathrm{~g} / \mathrm{cm}^{3}$.

The mass of a sample that has double-PP honeycomb is smaller than that of the single-PP honeycomb sample. The densities of the two samples indicate that their values are two times lower than that of polyvinyl chloride (PVC) (1.32-1.42 g/ $\mathrm{cm}^{3}$ ) [17]. This property gives a significant advantage for its application in a stroller.

\subsection{Creation of composite prototype}

After the positive result in terms of mechanical and physical properties, which were determined on the flat composite, the next step is to create a $3 \mathrm{D}$ composite prototype. The purpose of the prototype is to make the idea more realistic and tangible.

The prototype was formed using various materials, such as plastic bowl for molding, previously designed 3D woven fabric, and single-PP honeycomb layer, and implemented by applying the vacuum bagging method (Figure 12).

\subsubsection{Result of composite prototype}

The final prototype (Figure 13) is made up of a single-PP honeycomb sandwiched between two layers of flax fabric. It has the following dimensions: $18.5 \mathrm{~cm}$ width and $28.4 \mathrm{~cm}$ length, with a weight of $248 \mathrm{~g}$.

\section{Conclusion}

The intention of this project was to design and fabricate a 3D woven fabric as an element of a baby stroller. We developed an egg-shaped structure for the stroller seat using a woven fabric (natural flax fiber and PE) as a reinforcing material. For the composite preparation, to maximize the thickness of the composite, a PP honeycomb layer was added between two layers of woven reinforcing materials. The results of the tests on the mechanical and physical properties of the developed flat

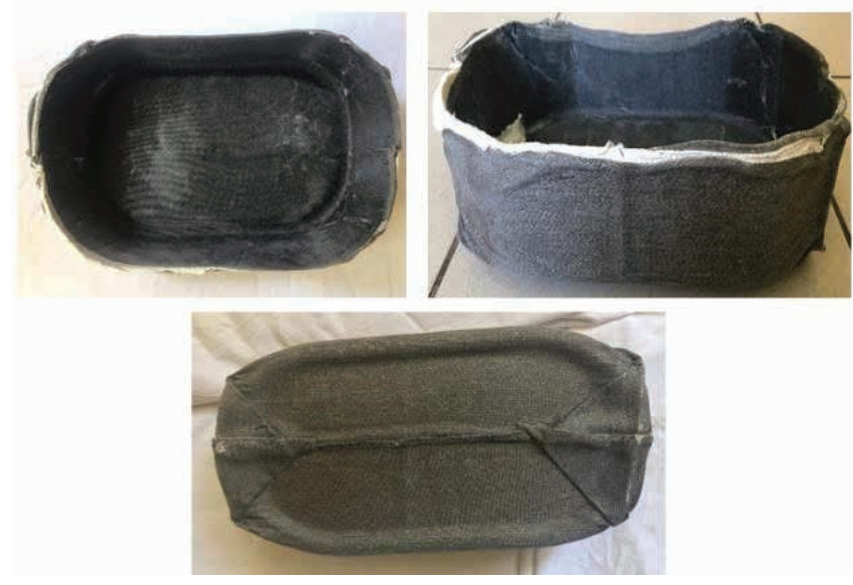

Figure 13. Final prototype result. 
composite gives a clue for the fabrication of $3 D$ shell-shaped composite structure, and the results are stated below:

- Depending on the tests for the mechanical properties, the tensile strength of the flat sample is greater than that of PVC by $26.06 \%$. But the bending strength of PVC is greater by $6.98 \%$ relative to the flax composite sample.

- The density of the flat composite sample is two times lower than that of PVC. This means that as the density decreases, the lightweight nature of the material increases, which means that the flat composite has favorable lightweight properties compared with PVC.

- The desired 3D shape is created by using a double-fabric weaving system using flax fiber as weft yarn, and using it as a reinforcing material, the 3D composite was formed.

Generally, the created composite possesses favorable properties, such as light weight and tensile strength. As described earlier, these can be comparable with the mechanical properties of PVC for the production of the stroller. In addition, the other advantage is that it is favorable to replace the PVC with a natural ecofriendly fabric that has enormous significance in relation to the health status of the consumers. To maximize the tensile properties of the composite the woven fabric has to be prepared with a flax yarn on both sides (weft and warp) directions. This research will be also continued by preparing a normal-sized stroller by using a $3 \mathrm{D}$ woven structure in the future.

\section{Acknowledgments}

The authors would like to extend our warmest and deepest gratitude to Mr. Henryk Browarsk for his effort and the manner in which he cooperated with us during the preparation of the samples. Moreover, we would like also to thank Dr. Mariusz Urbaniak, from the Faculty of Mechanical Engineering, for his consistent assistance during the laboratory testing process of the mechanical performance.

\section{References}

[1] Xiaogang, C. (2015). Advanced in 3D textiles. Woodhead Publishing.

[2] Pelin Gurkan Unal. (2012). "3D woven fabric." W woven fabric, Autor: Han-Yong Jeon, 91-120. Turkey: Intech-open publishing.

[3] Antonio, M. (1999). 3D textile reinforcement in composite materials. Woodhead Publishing.
[4] N. Khokar. (2001). 3D weaving; theory and practice. Journal of the Textile Institute, 92, 193-207.

[5] Oliver, D., Thomas, G., Chokri, C. (2014). Modelling of textile composite reinforcement on the micro-scale. AUTEX, 14(1). DOI: 10.2478/v10304-012-0047-z.

[6] Jinlian, H. (2008). 3D fibrous assemblies; properties, applications and modeling of three-dimensional textile structures. Woodhead Publishing (Cambridge, England).

[7] Hari, P. K., Behera, B. K. (2010). Woven textile structure,theory and applications. Woodhead Publishing (Cambridge).

[8] Barburski, M., Urbaniak, M., Samal, S. K. (2019). Comparison of mechanical properties of biaxial and triaxial fabric and composites reinforced by them. Fibers and textiles in Eastern Europe, 2019, 37-44.

[9] Kamińska, A., Barburski, M. (2018). 3D woven fabric with cross rib as a composite reinforcement. IOP Conference Series: Materials Science and Engineering, 460(2018), 012021 IOP Publishing. DOI: 10.1088/1757899X/460/1/012021.

[10] Barburski, M., Weigert, L., Fernández, I., Pouplier, S., Roth, S., Huurnink, G. (2017). Woven reinforced composites for improving the design of the hyperextension brace. Journal of Fashion Technology \& Textile Engineering, 2017, S3. DOI: 10.4172/2329-9568.S3-002.

[11] Dapeng, G., Yulin, Y., Suwen, C., Wenwen, S. (2014). A wear geometry model of plain-woven fabric composites. AUTEX, 14(3). DOI: 10.2478/aut-2014-0014.

[12] Vanleeuw B, Carvelli V, Lomov S.V, Barburski M, Vuure A.W. (2014). Deformability of a flax reinforcement for composite materials. Key Engineering Materials, 611612, 257-264; Trans Tech Publications, Switzerland. DOI: 10.4028/www.scientific.net/KEM.611-612.257.

[13] Evgeny, V. M. (2004). Mechanics and analysis of fabric composites and structures. AUTEX, 60-70.

[14] Nirmal, U., Jin, Z., Sehat, A. R. (2017). State of the art baby strollers. Cogent Engineering, 2017.

[15] Vanleeuw, B., Carvelli, V., Barburski, M., Lomov, S. V., Aart, W., van Vuure (2015). Quasi- unidirectional flax composite reinforcement: deformability and complex shape forming. Composites Science and Technology, 110, 76-86. DOI: 10.1016/j.compscitech.2015.01b.

[16] CorexHoneycomb. (2019). Web site: https:// corexhoneycomb.co.uk/products $\% 20$ and $\% 20$ services\%20/polypropylenehoneycomb/.

[17] Polyvinyl Chloride (PVC). (1998-2019). Retrieved from LENNTECH: https://www.lenntech.com/polyvinyl-chloridepvc.htm.

[18] CompositesUK. (2019). Retrieved from compositesuk. co.uk; https://compositesuk.co.uk/composite-materials/ processes.

[19] Vacuum Bagging Techniques. (2010). Gougeon Brothers (Bay City, MI, USA). 